\title{
BMJ Open Observational study of haemostatic dysfunction and bleeding in neonates with hypoxic-ischaemic encephalopathy
}

\author{
Mitali A Pakvasa, ${ }^{1,2}$ Anne M Winkler, ${ }^{3}$ Shannon E Hamrick, ${ }^{1,2}$ \\ Cassandra D Josephson, ${ }^{1,2,3}$ Ravi M Patel ${ }^{1,2}$
}

To cite: Pakvasa MA, Winkler AM, Hamrick SE, et al. Observational study of haemostatic dysfunction and bleeding in neonates with hypoxic-ischaemic encephalopathy. BMJ Open 2017;6:e013787.

doi:10.1136/bmjopen-2016013787

- Prepublication history and additional material is available. To view please visit the journal (http://dx.doi.org/ 10.1136/bmjopen-2016013787).

Received 6 August 2016 Revised 2 December 2016 Accepted 14 December 2016

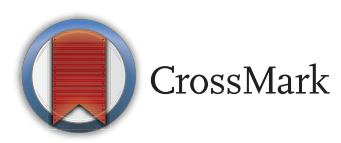

For numbered affiliations see end of article.

Correspondence to Dr Ravi M Patel; rmpatel@emory.edu

\section{ABSTRACT}

Objective: Evaluate the relationship between initial haemostatic parameters and the frequency and severity of bleeding in neonates with hypoxic-ischaemic encephalopathy (HIE).

Design: Retrospective observational cohort study.

Setting: 2 academically affiliated level III neonatal intensive care units in Atlanta, Georgia.

Participants: 98 neonates with moderate-to-severe HIE who underwent haemostatic testing within 12 hours of birth and were born from 1 January 2008 to 31 December 2013.

\section{Primary and secondary outcome measures:}

Initial haemostatic dysfunction was defined as one or more of the following: prothrombin time $(\mathrm{PT}) \geq 18 \mathrm{~s}$, platelet count $<100 \times 10^{3} / \mu \mathrm{L}$ or fibrinogen $<150 \mathrm{mg} / \mathrm{dL}$. Bleeding assessed using the Neonatal Bleeding Assessment Tool and graded according to the WHO bleeding scale. The robust Poisson regression was used to evaluate the independent association between components of initial haemostatic dysfunction and bleeding.

Results: Among the 98 neonates evaluated, the prevalence of initial haemostatic dysfunction was $69 \%$ (95\% Cl 59\% to $78 \%$ ). 27 neonates (28\%; 95\% Cl 19\% to $38 \%$ ) had abnormal bleeding events and $56(57 \%)$ received at least 1 blood product transfusion. 3 neonates died from bleeding complications. The most common products transfused were fresh-frozen plasma (71\%), followed by packed red blood cells $(24 \%)$ and platelets $(21 \%)$. In multivariable analysis, fibrinogen $<150 \mathrm{mg} / \mathrm{dL}$ (adjusted relative risk $2.41,95 \% \mathrm{Cl} 1.09$ to 5.36 ) and platelet count $<100 \times 10^{3} / \mu \mathrm{L}$ (adjusted relative risk $2.59,95 \% \mathrm{Cl} 1.30$ to 5.16 ), but not initial PT, were associated with an increased risk of bleeding. The most severe bleeding occurred in neonates with a fibrinogen $<150 \mathrm{mg} / \mathrm{dL}$.

Conclusions: Among neonates with moderate-tosevere HIE, haemostatic dysfunction is prevalent and associated with an increased risk of bleeding and high transfusion burden. Further studies are needed to determine the appropriate transfusion approaches in this population to prevent bleeding.

\section{INTRODUCTION}

Hypoxic-ischaemic encephalopathy (HIE) is a significant cause of neonatal morbidity and mortality and multiorgan dysfunction is

\section{Strengths and limitations of this study}

- This study estimated the incidence and quantified the location and severity of bleeding in neonates with hypoxic-ischaemic encephalopathy (HIE) using the NeoBAT, a standardised bleeding assessment tool for high-risk neonates.

- This study systematically evaluated the relationship between initial haemostatic dysfunction and the incidence, risk and severity of bleeding in infants with HIE.

- Study estimates of haemostatic dysfunction may be overestimated due to diagnostic bias, in which clinician-directed testing was more commonly performed in infants with greater illness severity.

- The association between initial haemostatic dysfunction and bleeding could have been biased by reverse causation, in which early bleeding resulted in haematological abnormalities.

common. ${ }^{2}$ Disruption of haemostasis can occur from ischaemic injury, acute blood loss or disseminated intravascular coagulation. ${ }^{3-6}$ In neonates with HIE enrolled in clinical trials of therapeutic hypothermia, the incidence of bleeding ranged from $3 \%$ to $12 \% .^{7} 8$ A more recent observational study reported a higher incidence of bleeding of $54 \%$ among neonates undergoing therapeutic hypothermia. ${ }^{5}$ In addition, the frequency of coagulopathy among neonates with HIE has also varied with reported prevalence ranging from $12 \%$ to $43 \% .^{7-9}$ Only one prior study has evaluated the association between coagulation parameters and abnormal bleeding events in this population. ${ }^{5}$ In this study, thresholds of platelet count $<130 \times 10^{3} / \mu \mathrm{L}$, fibrinogen activity $<1.5 \mathrm{~g} / \mathrm{L}$ and international normalised ratio (INR) $>2.0$ discriminated between neonates with and without bleeding. However, this study did not specifically evaluate the prevalence of initial haemostatic function. Additionally, no studies have graded the severity of 
bleeding in neonates with HIE and there are limited data to guide transfusion therapy, despite the common administration of blood products in this population.

Understanding haemostatic dysfunction among neonates with HIE may help guide risk assessment and transfusion practices in this high-risk population. We sought to estimate the prevalence of initial haemostatic dysfunction in neonates with moderate-to-severe HIE and to characterise the incidence, severity and location of bleeding. In addition, we evaluated the relationship between specific components of initial haemostatic dysfunction and the frequency and severity of bleeding events.

\section{METHODS}

Patient population

This retrospective cohort study was performed at two academically affiliated level III neonatal intensive care units in Atlanta, Georgia, USA (Grady Memorial Hospital, Emory University Hospital Midtown). All consecutively admitted neonates born between 1 January 2008 and 31 December 2013 were eligible for inclusion if they met the following three criteria: (1) birth weight $\geq 1800 \mathrm{~g}$; (2) moderate-to-severe HIE; (3) testing of any haemostatic parameter within 12 hours of birth. Exclusion criteria included no initial haemostatic testing or testing after 12 hours of life. Appropriate oversight and approval was obtained from the Emory University Institutional Review Board and Grady Memorial Hospital Research Oversight Committee. The study was reported according to the STrengthening the Reporting of OBservational studies in Epidemiology (STROBE) statement. ${ }^{10}$

\section{Definitions}

Haemostatic dysfunction was defined as the presence of coagulopathy, thrombocytopenia or hypofibrinogenaemia (defined below) occurring within 12 hours of birth. Reagents used by the hospital coagulation laboratory did not have neonatal-specific reference ranges. Therefore, we selected a prothrombin time (PT) of $18 \mathrm{~s}$, a priori, to define coagulopathy, taking into consideration the values from published normative ranges, ${ }^{11}$ with some allowance for mild elevation. We chose to use PT as opposed to the INR to define coagulopathy, as INR was inconsistently reported or recorded at study sites. We also evaluated the sensitivity of using a cut-point by evaluating PT prolongation as a continuous variable. Thrombocytopenia was defined as a platelet count $<100 \times 10^{3} / \mu \mathrm{L}$ based on this value as the trigger for the upper threshold for platelet transfusion among neonatologists. ${ }^{12} 13$ Hypofibrinogenaemia was defined using the cut-point of $<150 \mathrm{mg} / \mathrm{dL}$, based on this value being the lower limit of normative ranges in healthy term newborns at birth and recently published data among neonates with HIE receiving therapeutic hypothermia. ${ }^{5}$ Haemostatic parameters outside of the diagnostic range were assigned to the upper or lower value of the detection limit. Moderate-to-severe HIE was determined using criteria from Neonatal Research Network trials. ${ }^{8}$ We evaluated all blood products transfused during the first 96 hours after birth. The decision to transfuse was at the decision of the treating neonatal caregivers, as the hospitals in this study did not have transfusion protocols or guidelines for this population of infants. Outcomes were compared between those neonates who experienced an abnormal bleeding event versus those who did not. We ascertained bleeding events during the first 96 hours after birth based on a diagnosis of or notation of abnormal bleeding in physician, nursing or respiratory therapist documentation. We also adapted the Neonatal Bleeding Assessment Tool (NeoBAT), a validated bleeding assessment tool, to retrospectively assess the severity of abnormal bleeding events. ${ }^{14}$ We used the modified NeoBAT to ascribe all patients with bleeding events a modified WHO bleeding assessment scale score (scale of $1-4$, with 4 being the most severe). Patients without bleeding were assigned a score of 0 .

\section{Statistical analysis}

SPSS 22.0 for Windows (IBM, Armonk, New York, USA) was used for all statistical analysis. Data acquisition and statistical analysis was performed from 22 October 2014 to 6 May 2016. As this study was a convenience sample, no sample size estimation was performed. Normally distributed continuous variables were described using means and SDs and compared using the Student's t-test. Medians with IQRs were used for non-normally distributed variables and compared using the Wilcoxon rank-sum tests. Categorical variables were compared using the $\chi^{2}$ or Fisher's exact tests. Binomial CIs were obtained using the Clopper and Pearson method. Denominators were specified to indicate missing data, when present; imputation was not performed. Relationships between PT, fibrinogen, platelet count and bleeding severity were characterised using a bubble plot. Linear trends between ordinal categories and response variables were evaluated using generalised linear models. The ability of initial coagulation parameters to discriminate bleeding and non-bleeding neonates was determined using standard measures of test performance with clinically determined and previously published cut-points. ${ }^{5}$ We also evaluated the performance of parameters in multivariable logistic regression models to discriminate bleeding versus non-bleeding neonates using the area under the curve.

The frequency of abnormal bleeding events was compared by the individual components of initial haemostatic dysfunction. The Poisson regression with robust SEs was used to evaluate the association between initial haemostatic dysfunction and abnormal bleeding events. Baseline haemostatic parameters were included in multivariable regression models, to determine the independent association of bleeding with individual haemostatic parameters. Only main effects were specified in the model. No interaction testing was performed in order to limit parameters 
Table 1 Maternal and neonatal characteristics

\begin{tabular}{|c|c|c|c|}
\hline Maternal characteristics & $\begin{array}{l}\text { No bleeding } \\
\mathrm{n}=71\end{array}$ & $\begin{array}{l}\text { Bleeding } \\
\mathrm{n}=27\end{array}$ & p Value \\
\hline Maternal age (years) & $28 \pm 7$ & $31 \pm 5$ & 0.03 \\
\hline Race & & & 0.96 \\
\hline White & 20/69 (29\%) & $6 / 24(25 \%)$ & \\
\hline Black & $34 / 69$ (49\%) & $12 / 24(50 \%)$ & \\
\hline Hispanic & $11 / 69(16 \%)$ & $4 / 24(17 \%)$ & \\
\hline Other & $4 / 69(6 \%)$ & $2 / 24(8 \%)$ & \\
\hline Caesarean delivery & $52 / 71(73 \%)$ & $22 / 27(81 \%)$ & 0.40 \\
\hline Severe trauma & $1 / 71(1 \%)$ & $0 / 27(0 \%)$ & $>0.99$ \\
\hline Placental abruption & $14 / 71(20 \%)$ & $9 / 27(33 \%)$ & 0.16 \\
\hline Uterine rupture & $6 / 71(8 \%)$ & $6 / 27(22 \%)$ & 0.09 \\
\hline Shoulder dystocia & $2 / 70(3 \%)$ & $1 / 27(4 \%)$ & $>0.99$ \\
\hline Neonatal characteristics & No bleeding & Bleeding & p Value \\
\hline Birth weight $(\mathrm{g})$ & $3200 \pm 606$ & $3390 \pm 690$ & 0.19 \\
\hline Gestational age (weeks) & $38.7 \pm 1.7$ & $38.9 \pm 1.7$ & 0.69 \\
\hline Female & $29 / 71(41 \%)$ & $11 / 27(41 \%)$ & 0.99 \\
\hline Inborn & $52 / 71(73 \%)$ & $18 / 27(67 \%)$ & 0.52 \\
\hline 1 min Apgar, median (IQR) & $1(0-2)$ & $1(0-1)$ & 0.23 \\
\hline 5 min Apgar, median (IQR) & $3(1-4)$ & $3(0-4)$ & 0.31 \\
\hline \multicolumn{4}{|l|}{ Delivery room care } \\
\hline Intubation & $64 / 70(91 \%)$ & $23 / 27(85 \%)$ & 0.46 \\
\hline Chest compressions & $32 / 70(46 \%)$ & $16 / 27(59 \%)$ & 0.23 \\
\hline Epinephrine & 23/70 (33\%) & $14 / 27(52 \%)$ & 0.08 \\
\hline Saline administration & $13 / 70(19 \%)$ & $11 / 27(41 \%)$ & 0.02 \\
\hline RBC administration & $0 / 70(0 \%)$ & $0 / 27(0 \%)$ & $>0.99$ \\
\hline Cord blood gas pH & $6.88 \pm 0.21$ & $6.85 \pm 0.19$ & 0.67 \\
\hline Admission blood gas $\mathrm{pH}$ & $7.03 \pm 0.23$ & $6.99 \pm 0.22$ & 0.52 \\
\hline \multicolumn{4}{|l|}{ Severity of HIE } \\
\hline Moderate & 49/71 (69\%) & $14 / 27(52 \%)$ & 0.11 \\
\hline Severe & 22/71 (31\%) & $13 / 27(48 \%)$ & \\
\hline Therapeutic hypothermia & 70/71 (99\%) & $27 / 27$ (100\%) & $>0.99$ \\
\hline Death (in hospital) & $8 / 71(11 \%)$ & $6 / 27(22 \%)$ & 0.20 \\
\hline Death from haemorrhage & $0 / 71(0 \%)$ & $3 / 27(11 \%)$ & 0.02 \\
\hline
\end{tabular}

Values listed are $\mathrm{n} / \mathrm{N}(\%)$ or mean $\pm \mathrm{SD}$, unless indicated otherwise.

Missing values for continuous variables: maternal age, 6; gestational age, 1; 1 min Apgar, 2; 5 min Apgar, 2; cord blood gas pH, 39; admission blood gas $\mathrm{pH}, 14$.

HIE, hypoxic-ischaemic encephalopathy; RBC, red blood cell.

to 1 per 10 outcome events to protect against overfitting. Because of the right-tailed distribution of PT and significant correlation between PT, platelet count and fibrinogen (Spearman's correlation coefficients of 0.44-0.54), we specified these values as categorical variables in the multivariable model. PT was categorised using approximate tertiles, with the first tertile consistent with our prespecified definition of coagulopathy. Activated partial thromboplastin time (APTT) and INR were not included, given the collinearity with PT.

\section{RESULTS}

Over a 72-month period, we reviewed medical records of 505 neonates who weighed $1800 \mathrm{~g}$ or more at birth and had either a 5 min APGAR $\leq 7$ or admission diagnosis of HIE. Of these, 132 were diagnosed with moderate-to-severe $\mathrm{HIE}$ and 98 neonates underwent haemostatic testing within 12 hours of birth and were included in the study for further analysis. Of note, we found no difference in the frequency of severe HIE ( $36 \%$ vs $29 \%, \mathrm{p}=0.50)$, bleeding ( $28 \%$ vs $24 \%$; $=0.65)$ or receipt of therapeutic hypothermia (98\% vs $94 \%$, $\mathrm{p}=0.16$ ) between neonates who underwent initial haemostatic testing within 12 hours of birth and were included in the study $(n=98)$ and those neonates who did not meet this inclusion criterion $(n=34)$.

\section{Patient characteristics}

We compared neonates with and without abnormal bleeding events. There were no significant differences in the mean birth weight or gestational age between neonates with and without bleeding (table 1). There were no significant differences in sex, hospital of birth, severity of HIE or 1 and 5 min Apgar scores between groups. Neonates with bleeding, compared with non-bleeding neonates, were more likely to have received saline 
during delivery room resuscitation $(41 \%$ vs $19 \%$, $\mathrm{p}=0.02)$. There was also no significant difference in maternal trauma, uterine rupture, shoulder dystocia or maternal placental abruption between neonates with and without bleeding. Ninety-nine per cent of neonates in the study received therapeutic hypothermia.

\section{Coagulation abnormalities and transfusion burden}

The prevalence of initial haemostatic dysfunction was $69 \%$ (95\% CI $59 \%$ to $78 \%$ ), largely accounted for by the high prevalence of a prolonged PT $\geq 18 \mathrm{~s}$. Neonates with bleeding had significantly higher initial PT, APTT and INR values, and lower fibrinogen activity platelet counts than neonates without bleeding (table 2). The most common blood products transfused in the first 96 hours after birth were fresh-frozen plasma, followed by packed red blood cells (table 2). Although the receipt of any blood product transfusion was more common among neonates with bleeding, approximately half of neonates without bleeding received at least one blood product transfusion. Platelet transfusion occurred only among neonates with bleeding.

\section{Bleeding outcomes}

Among the $28 \%$ (95\% CI $19 \%$ to $38 \%$ ) of neonates with abnormal bleeding, the most common sites of bleeding were pulmonary (23\%), upper gastrointestinal (21\%) and umbilical (18\%) (see online supplementary figure S1). Three neonates had haemorrhage as a contributing cause of death (table 1). Two of these neonates had refractory shock. One of these neonates had a large and expanding subgaleal bleed and the second neonate had a decrease in haematocrit of $19 \%$ within 24 hours and required massive transfusion. On autopsy, this second infant was found to have multiple areas of intra-abdominal haemorrhage (spleen, adrenal, liver, ileum). A third patient had significant pulmonary haemorrhage with respiratory deterioration, despite increased positive-end expiratory pressure and endotracheal epinephrine.

The per cent of neonates with abnormal bleeding events and the receipt of any blood products increased with increasing PT prolongation $(\mathrm{p}<0.001$ for linear trend for both comparisons) (see online supplementary figure S2). Of the 27 patients with an abnormal bleeding event, $10(37 \%)$ patients had a WHO bleeding severity score of $1,7(26 \%)$ had a score of $2,5(19 \%)$ had a score of 3 and $5(19 \%)$ a score of 4 . The WHO bleeding severity increased with increasing PT and decreasing fibrinogen levels. Twenty per cent of infants with a bleeding severity score of 3 had an initial platelet count $<100 \times 10^{3} / \mu \mathrm{L}$ and none of the infants with bleeding severity score of 4 had an initial platelet count $<100 \times 10^{3} / \mu \mathrm{L}$ (figure 1 ).

\section{Risk factors for bleeding}

In a bivariable analysis, the risk of bleeding increased by $22 \%$ for every $10 \mathrm{~s}$ increase in initial PT (relative risk $1.22 ; 95 \%$ CI 1.15 to 1.30 ) (table 3 ). In multivariable analysis, fibrinogen $<150 \mathrm{mg} / \mathrm{dL}$ (adjusted relative risk $2.41,95 \%$ CI 1.09 to 5.36$)$ and platelet count $<100 \times 10^{3} / \mu \mathrm{L}$ (adjusted relative risk 2.23, 95\% CI 1.17 to 4.22 ), but not degree of PT prolongation, were independently associated with abnormal bleeding. Results were similar when evaluating neonates with a fibrinogen $<100 \mathrm{mg} / \mathrm{dL}$ instead of $<150 \mathrm{mg} / \mathrm{dL}$.

Among initial haemostatic parameters evaluated, an initial PT $\geq 18 \mathrm{~s}$ had the highest sensitivity (88\%) and lowest specificity (38\%) for discriminating neonates with and without abnormal bleeding, while an initial platelet count $<100 \times 10^{3} / \mu \mathrm{L}$ had the highest specificity $(96 \%)$ and lowest sensitivity (27\%) (see online supplementary table S1). We also evaluated the ability of previously published cut-points ${ }^{5}$ to discriminate bleeding in our cohort and found similar performance characteristics for fibrinogen $<154 \mathrm{mg} / \mathrm{dL}$, but not platelet count $<130.5 \times 10^{3} / \mu \mathrm{L}$ or INR $>1.98$.

\section{DISCUSSION}

Initial haemostatic dysfunction is highly prevalent among neonates with moderate-to-severe HIE, and is associated with a high transfusion burden and increased risk of bleeding. The prevalence of haemostatic dysfunction in our cohort was $69 \%$, which is higher than the $18-54 \%$ reported in prior published studies. ${ }^{5-9}$ Some of the prior studies used a more restrictive definition of coagulopathy, which included the need for clinical bleeding, potentially resulting in underestimation of the actual prevalence of haemostatic dysfunction. Our results demonstrate blood product transfusions with packed red blood cells, platelets and fresh-frozen plasma are common in neonates with moderate-to-severe HIE, among bleeding and non-bleeding neonates.

Transfusions are life saving for certain neonates, but there are no standard guidelines for transfusion practices in term neonates with significant variation among medical providers. ${ }^{12} 13$ Early correction of haemostatic dysfunction with massive transfusion protocols has been associated with improved outcomes in paediatric trauma patients. $^{15} 16$ Although the mechanisms of haemostatic dysfunction in neonates with HIE differ from paediatric trauma patients, they may benefit from a standardised transfusion protocol, given the common utilisation of multiple blood product types. Given the strong association between the severity of haemostatic dysfunction and bleeding risk, we believe that prophylactic transfusions with blood products based on laboratory data alone are reasonable. However, the appropriate transfusion thresholds and whether prophylactic transfusion at these thresholds can prevent serious bleeding is uncertain in this population.

We found that thrombocytopenia and hypofibrinogenaemia were independently associated with an increased risk of bleeding. Neonates with lower WHO bleeding scale scores ( 1 or 2 ) were more likely to have thrombocytopenia compared with neonates with more severe 
Table 2 Haemostatic and transfusion characteristics among bleeding and non-bleeding neonates

\begin{tabular}{|c|c|c|c|}
\hline Haemostatic characteristics & $\begin{array}{l}\text { No bleeding } \\
\mathrm{n}=71\end{array}$ & $\begin{array}{l}\text { Bleeding } \\
\mathrm{n}=27\end{array}$ & p Value \\
\hline PT (s) (n=95) & $19(17-26)$ & $28(18-58)$ & 0.004 \\
\hline $\operatorname{APTT}(s)(n=74)$ & $54(45-63)$ & $72(51-135)$ & 0.005 \\
\hline INR $(n=82)$ & $1.7(1.4-2.4)$ & $2.7(1.6-4.4)$ & 0.008 \\
\hline Fibrinogen $(m g / d L)(n=83)$ & $163(135-199)$ & $80(70-141)$ & $<0.001$ \\
\hline Initial platelet count $\left(10^{3} / \mu \mathrm{L}\right)(\mathrm{n}=93)$ & $190(160-235)$ & $156(98-174)$ & 0.001 \\
\hline $\mathrm{PT} \geq 18 \mathrm{~s}$ & $43 / 69(62 \%)$ & $23 / 26(89 \%)$ & 0.01 \\
\hline Fibrinogen $<100 \mathrm{mg} / \mathrm{dL}$ & $7 / 61(12 \%)$ & $13 / 22(59 \%)$ & $<0.001$ \\
\hline Fibrinogen $<150 \mathrm{mg} / \mathrm{dL}$ & $22 / 61(36 \%)$ & $17 / 22(77 \%)$ & 0.001 \\
\hline Initial platelet count $<100 \times 10^{3} / \mu \mathrm{L}$ & $3 / 67(5 \%)$ & $7 / 26(27 \%)$ & 0.004 \\
\hline \multicolumn{4}{|l|}{ Transfusion characteristics } \\
\hline \multicolumn{4}{|l|}{ Frequency of blood product transfusions } \\
\hline \multicolumn{4}{|l|}{ Packed red blood cell } \\
\hline None & $58 / 71(82 \%)$ & $17 / 27(63 \%)$ & 0.049 \\
\hline 1 & $10 / 71(14 \%)$ & $5 / 27(19 \%)$ & \\
\hline 2 or greater & $3 / 71(4 \%)$ & $5 / 27(19 \%)$ & \\
\hline \multicolumn{4}{|l|}{ Platelet } \\
\hline None & $71 / 71(100 \%)$ & $20 / 27(74 \%)$ & $<0.001$ \\
\hline 1 & $0 / 71(0 \%)$ & $3 / 27(11 \%)$ & \\
\hline 2 or greater & $0 / 71(0 \%)$ & $4 / 27(15 \%)$ & \\
\hline \multicolumn{4}{|l|}{ Fresh-frozen plasma } \\
\hline None & $41 / 71(58 \%)$ & 9/27 (33\%) & 0.002 \\
\hline 1 & $16 / 71(23 \%)$ & $3 / 27(11 \%)$ & \\
\hline 2 or greater & $14 / 71(20 \%)$ & $15 / 27(56 \%)$ & \\
\hline \multicolumn{4}{|l|}{ Cryoprecipitate } \\
\hline None & 70/71 (99\%) & $24 / 27(90 \%)$ & 0.08 \\
\hline 1 & $1 / 71(1 \%)$ & $2 / 27(7 \%)$ & \\
\hline 2 or greater & $0 / 71(0 \%)$ & $1 / 27(4 \%)$ & \\
\hline Any blood product transfusion & $36 / 71(51 \%)$ & $20 / 27$ (74\%) & 0.04 \\
\hline
\end{tabular}

Figure 1 Relationship between haemostatic parameters and bleeding severity. Bubble plot demonstrates initial haemostatic parameters among 97 patients. The size of the bubbles increases with increasing bleeding severity, determined using the $\mathrm{WHO}$ bleeding scale and ascertained using the Neonatal Bleeding Assessment Tool (NeoBAT). One infant with an initial fibrinogen of $1400 \mathrm{mg} / \mathrm{dL}$ and bleeding score of 2 not shown. The frequency of abnormal haemostatic parameters by bleeding severity score is reported in the inset box.

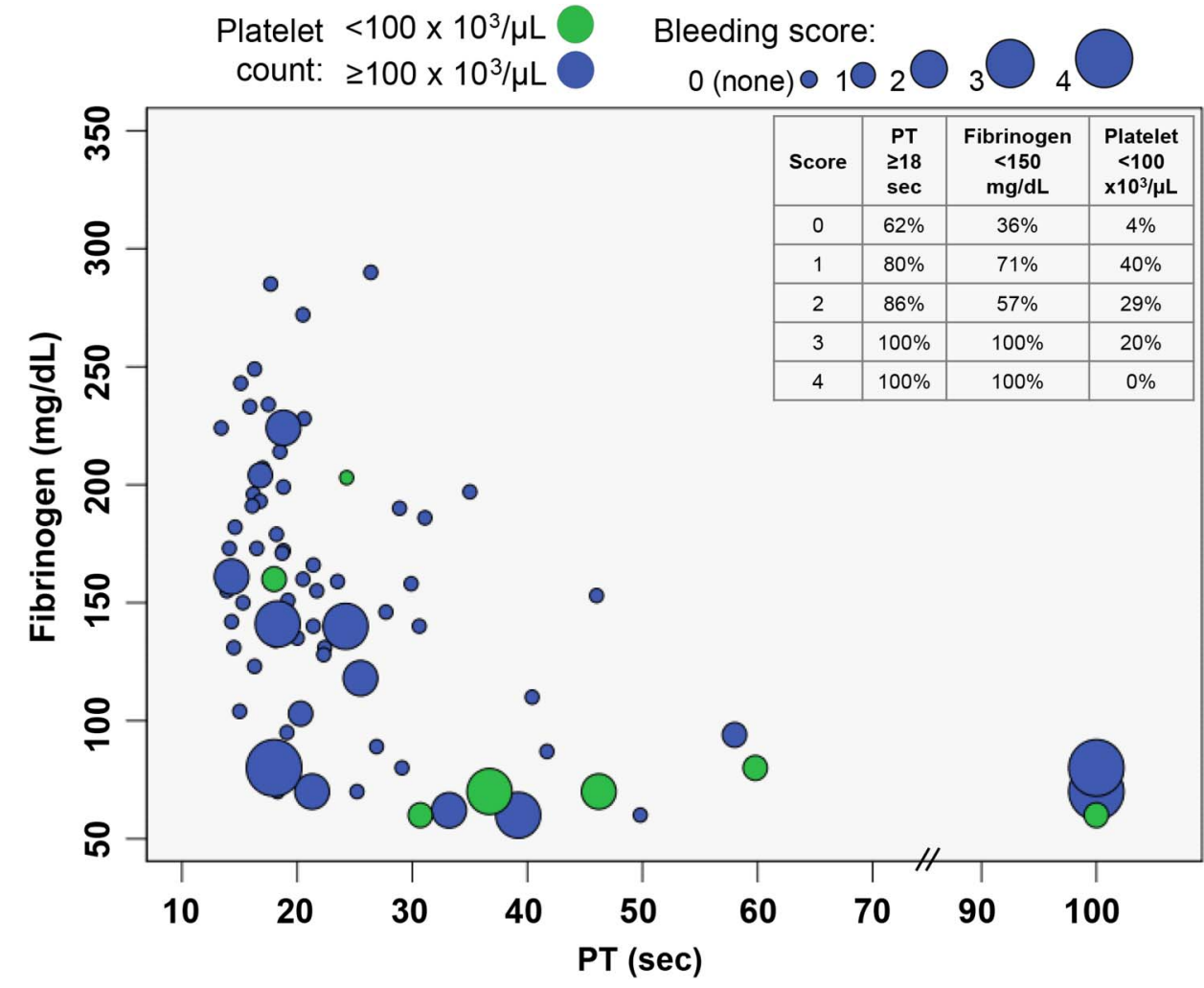


Table 3 Bivariable and multivariable risk factors for abnormal bleeding

\begin{tabular}{|c|c|c|c|c|}
\hline Factor (bivariable) & $\mathrm{n} / \mathrm{N}$ & $\mathbf{R R}$ & $95 \% \mathrm{Cl}$ & p Value \\
\hline \multicolumn{5}{|l|}{ PT (categorised) } \\
\hline$<18 \mathrm{~s}$ & 29/95 & 1.00 & Reference & \\
\hline $18-30 \mathrm{~s}$ & $42 / 95$ & 2.30 & 0.69 to 7.64 & 0.17 \\
\hline 30 or greater s & $24 / 95$ & 5.24 & 1.69 to 16.3 & 0.004 \\
\hline PT (per $10 \mathrm{~s}$ increase) & 95 & 1.22 & 1.15 to 1.30 & $<0.001$ \\
\hline APTT (per 10 s increase) & 74 & 1.08 & 1.05 to 1.12 & $<0.001$ \\
\hline INR (per 1 point increase) & 82 & 1.17 & 1.06 to 1.30 & 0.003 \\
\hline Fibrinogen $<100 \mathrm{mg} / \mathrm{dL}$ & $20 / 83$ & 4.55 & 2.29 to 9.03 & $<0.001$ \\
\hline Fibrinogen $<150$ mg/dL & $39 / 83$ & 3.84 & 1.56 to 9.43 & 0.003 \\
\hline Platelet $<100 \times 10^{3} / \mu \mathrm{L}$ & $10 / 93$ & 3.06 & 1.74 to 5.39 & $<0.001$ \\
\hline Initial pH (per 1 point increase) & 84 & 0.62 & 0.16 to 2.38 & 0.49 \\
\hline HIE (severe vs moderate) & $35 / 98$ & 1.67 & 0.89 to 3.14 & 0.11 \\
\hline Placental abruption & $23 / 98$ & 1.63 & 0.85 to 3.12 & 0.14 \\
\hline Uterine rupture & $12 / 98$ & 2.05 & 1.04 to 4.03 & 0.04 \\
\hline Factor (multivariable)* & & Adjusted RR & $95 \% \mathrm{Cl}$ & p Value \\
\hline \multicolumn{5}{|l|}{ PT (categorised) } \\
\hline$<18 \mathrm{~s}$ & $23 / 77$ & 1.00 & Reference & \\
\hline 18 to $<30 \mathrm{~s}$ & $36 / 77$ & 1.77 & 0.43 to 7.29 & 0.43 \\
\hline 30 or greater s & $18 / 77$ & 2.69 & 0.65 to 11.1 & 0.17 \\
\hline Fibrinogen $<150$ mg/dL & $36 / 77$ & 2.41 & 1.09 to 5.36 & 0.03 \\
\hline Platelet $<100 \times 10^{3} / \mu \mathrm{L}$ & $8 / 77$ & 2.59 & 1.30 to 5.16 & 0.007 \\
\hline
\end{tabular}

${ }^{*}$ For multivariable robust Poisson regression model, only 77 of 98 patients with all initial haemostatic parameters measured were included. Area under the curve of $0.81(95 \% \mathrm{Cl} 0.69$ to 0.93$)$, obtained from multivariable logistic regression model.

APTT, activated partial thromboplastim time; HIE, hypoxic-ischaemic encephalopathy; INR, international normalised ratio; PT, prothrombin time; RR, relative risk.

bleeding, suggesting a disruption of primary haemostasis. In contrast, coagulopathy and hypofibrinogenaemia were more common among neonates with more severe bleeding (scores of 3 or 4), suggesting a problem of secondary haemostasis. Although platelet count $<100 \times 10^{3} /$ $\mu \mathrm{L}$ was a predictor of abnormal bleeding, most patients with bleeding had platelet counts $>100 \times 10^{3} / \mu \mathrm{L}$. In the setting of moderate-to-severe HIE, where decreased $\mathrm{pH}$ and hypothermia are present, platelet dysfunction despite a normal platelet count may impact the risk of bleeding. The use of cryoprecipitate was relatively infrequent in our study, despite the common finding of hypofibrinogenaemia, particularly among neonates with higher bleeding severity. This contrasts the nearly $40 \%$ use of cryoprecipitate in another report. ${ }^{5}$ Cryoprecipitate has a higher fibrinogen content than fresh-frozen plasma. Additional studies are necessary to determine whether early cryoprecipitate use is superior to fresh-frozen plasma to prevent bleeding in this population. In addition, our study highlights the importance of systematically evaluating neonates with HIE for initial haemostatic dysfunction, given $74 \%$ of infants received initial haemostatic evaluation, despite adherence to whole body hypothermia protocols that were continued following participation by the two study hospitals in the initial hypothermia trials by the Neonatal Research Network. Of note, approximately half the infants excluded had initial haemostatic evaluation performed after 12 hours of age. Initial haemostatic testing can help identify neonates at higher risk for bleeding complications who could potentially benefit from prophylactic blood product transfusions. Further studies are needed to evaluate transfusion practices, including the relationship between cryoprecipitate transfusion versus fresh-frozen plasma transfusion on bleeding risk and other important outcomes among neonates with moderate-to-severe HIE. Given many of the infants with bleeding received multiple blood products, the role of balanced ratios of transfusion in this population may warrant additional study.

Our study has several strengths. We were able to evaluate a relatively large number of patients at two centres, given our large referral base for neonates with HIE. We were able to quantify bleeding in our patients using the NeoBAT, a standardised bleeding assessment tool for high-risk neonates with high inter-rater reliability when applied in the clinical setting. In addition, this is the first study that has systematically evaluated initial haemostatic dysfunction in patients with moderate-to-severe HIE and the relationship between these parameters and the severity of bleeding.

Our study has several limitations. Since this was a retrospective study, we relied on clinician-ordered haemostatic testing and not all neonates underwent laboratory testing or had testing of all haemostatic parameters. As a result, our results may be biased because haemostatic testing could have been more commonly obtained in neonates with clinical evidence of 
multisystem organ dysfunction or greater illness severity. However, we found no difference in the severity of HIE, receipt of therapeutic hypothermia or frequency of bleeding between neonates with and without baseline haemostatic testing. In addition, it is possible that haemostatic dysfunction could have been the result of bleeding. To address this, we only included testing that was performed within the first 12 hours of birth and we only evaluated bleeding events for the first 96 hours after birth to reduce the possibility that bleeding was the cause of haemostatic dysfunction or temporally unrelated to initial haematological testing. In addition, haematological parameters in some neonates were obtained following the start of therapeutic hypothermia. However, a prior study suggests this is unlikely to have a significant effect on coagulation parameters. ${ }^{17}$ Furthermore, we could not prospectively characterise bleeding in each infant, which may limit the utility of the NeoBAT in ascertaining bleeding severity.

In conclusion, initial haemostatic dysfunction is prevalent among patients with moderate-to-severe HIE and the severity of haemostatic dysfunction is associated with an increased risk of the frequency and severity of bleeding. These findings indicate that assessment of initial haemostatic dysfunction should be performed routinely in all neonates with moderate-to-severe HIE.

\section{Author affiliations}

${ }^{1}$ Department of Pediatrics, Emory University School of Medicine, Atlanta, Georgia, USA

${ }^{2}$ Children's Healthcare of Atlanta, Atlanta, Georgia, USA

${ }^{3}$ Pathology and Laboratory Medicine, Emory University School of Medicine, Atlanta, Georgia, USA

\section{Twitter Follow Ravi Patel @ravimpatelmd}

Contributors MAP conceived the study, acquired, analysed and interpreted the data, wrote the first draft of the manuscript and approved the final submitted version. AMW interpreted the data, revised the manuscript for important intellectual content and approved the final submitted version. SEH was involved in the study design, interpreted the data, revised the manuscript for important intellectual content and approved the final submitted version. CDJ was involved in the study design, interpreted the data, revised the manuscript for important intellectual content and approved the final submitted version. RMP conceived the study, analysed and interpreted the data, revised the manuscript for important intellectual content and approved the final submitted version. All authors agree to be accountable for the accuracy and integrity of the work.

Funding The National Institutes of Health $(\mathrm{NIH})$ under award numbers UL1 TR000454, KL2 TR000455 and K23 HL128942 provided support for RMP.

Disclaimer The NIH had no role in: (1) study design; (2) the collection, analysis, and interpretation of data; (3) the writing of the report; and (4) the decision to submit the paper for publication.

Competing interests AMW accepted employment by Instrumentation Laboratory following the conclusion of the study. Instrumentation Laboratory is involved in diagnostic instruments for haemostasis. No company products were used in the testing for this study. CDJ serves as a consultant for
Immocur and Zimmer Biomet. Neither company had any role in this study. The remaining authors have no competing interests to disclose.

Ethics approval Appropriate oversight and approval was obtained from the Emory University Institutional Review Board and Grady Memorial Hospital Research Oversight Committee (IRB\# 00075975).

Provenance and peer review Not commissioned; externally peer reviewed.

Data sharing statement Extra data can be accessed via the Dryad data repository at http://datadryad.org/ with the doi:10.5061/dryad.gn5c8.

Open Access This is an Open Access article distributed in accordance with the terms of the Creative Commons Attribution (CC BY 4.0) license, which permits others to distribute, remix, adapt and build upon this work, for commercial use, provided the original work is properly cited. See: http:// creativecommons.org/licenses/by/4.0/

\section{REFERENCES}

1. Azzopardi D, Strohm B, Marlow N, et al. Effects of hypothermia for perinatal asphyxia on childhood outcomes. $N$ Engl J Med 2014;371:140-9.

2. Shankaran S, Pappas A, McDonald SA, et al. Childhood outcomes after hypothermia for neonatal encephalopathy. $N$ Engl $J$ Med 2012;366:2085-92.

3. Shah P, Riphagen S, Beyene J, et al. Multiorgan dysfunction in infants with post-asphyxial hypoxic-ischaemic encephalopathy. Arch Dis Child Fetal Neonatal Ed 2004;89:F152-5.

4. Bauman ME, Cheung PY, Massicotte MP. Hemostasis and platelet dysfunction in asphyxiated neonates. J Pediatr 2011;158(Suppl 2): e35-9.

5. Forman KR, Diab Y, Wong EC, et al. Coagulopathy in newborns with hypoxic ischemic encephalopathy (HIE) treated with therapeutic hypothermia: a retrospective case-control study. BMC Pediatr 2014;14:277.

6. Suzuki S, Morishita S. Hypercoagulability and DIC in high-risk infants. Semin Thromb Hemost 1998;24:463-6.

7. Gluckman PD, Wyatt JS, Azzopardi D, et al. Selective head cooling with mild systemic hypothermia after neonatal encephalopathy: multicentre randomised trial. Lancet 2005;365:663-70.

8. Shankaran S, Laptook AR, Ehrenkranz RA, et al. Whole-body hypothermia for neonates with hypoxic-ischemic encephalopathy. N Engl J Med 2005;353:1574-84.

9. Azzopardi DV, Strohm B, Edwards AD, et al. Moderate hypothermia to treat perinatal asphyxial encephalopathy. $N$ Engl $J$ Med 2009;361:1349-58.

10. von Elm E, Altman DG, Egger M, et al. The strengthening the reporting of observational studies in epidemiology (STROBE) statement: guidelines for reporting observational studies. Prev Med 2007; 45:247-51.

11. Andrew M, Paes B, Milner R, et al. Development of the human coagulation system in the full-term infant. Blood 1987;70:165-72.

12. Josephson CD, Su LL, Christensen RD, et al. Platelet transfusion practices among neonatologists in the United States and Canada: results of a survey. Pediatrics 2009;123:278-85.

13. Venkatesh $\mathrm{V}$, Khan R, Curley $\mathrm{A}$, et al. How we decide when a neonate needs a transfusion. Br J Haematol 2013;160:421-33.

14. Venkatesh V, Curley A, Khan R, et al. A novel approach to standardised recording of bleeding in a high risk neonatal population. Arch Dis Child Fetal Neonatal Ed 2013;98:F260-3.

15. Hendrickson JE, Shaz BH, Pereira G, et al. Coagulopathy is prevalent and associated with adverse outcomes in transfused pediatric trauma patients. J Pediatr 2012;160:204-9 e3.

16. Hendrickson JE, Shaz BH, Pereira G, et al. Implementation of a pediatric trauma massive transfusion protocol: one institution's experience. Transfusion 2012;52:1228-36.

17. Sarkar S, Barks JD, Bhagat I, et al. Effects of therapeutic hypothermia on multiorgan dysfunction in asphyxiated newborns: whole-body cooling versus selective head cooling. J Perinatol 2009;29:558-63. 